Published in final edited form as:

Surg Obes Relat Dis. 2013 January ; 9(1): 94-99. doi:10.1016/j.soard.2011.10.014.

\title{
NECDIN-E2F4 INTERACTION PROVIDES INSULIN-SENSITIZING EFFECT AFTER WEIGHT LOSS INDUCED BY GASTRIC BYPASS SURGERY
}

\author{
Pamuklar Zehra N ${ }^{1}$, Chen Jie-gen ${ }^{1}$, Muehlbauer Michael ${ }^{2}$, Spagnoli Anna ${ }^{3}$, and Torquati \\ Alfonso $^{1,2}$ \\ ${ }^{1}$ Department of Surgery, Duke University, USA \\ ${ }^{2}$ Sarah W. Stedman Nutrition and Metabolism Center, Duke University, USA \\ ${ }^{3}$ Department of Pediatrics, University of North Carolina, USA
}

\section{Abstract}

Background-The insulin/ growth factor 1 (IGF-1) signaling pathway promotes adipocyte differentiation and therefore insulin sensitivity via suppression of Necdin expression, which represses PPAR $\gamma$ promoter activity via interaction with $\mathrm{E} 2 \mathrm{~F} 4$ in mouse adipocytes.

Objectives-The aim of this study was to test the hypothesis that this pathway represents one of the mechanisms by which Roux-en-Y gastric bypass surgery (RYGB) induces resolution of insulin resistance.

\begin{abstract}
Methods-Clinical samples were collected and key biomarkers were measured in order to test the hypothesis that the IGF-1 pathway represents one of the mechanisms by which RYGB induces resolution of insulin resistance in obese individuals.
\end{abstract}

Results-Free IGF-1 levels were significantly higher in the post-RYGB patients as compared to pre-RYGB obese patients $(2.55 \pm 1.54$ vs. $1.32 \pm 0.65 \mathrm{mcg} / \mathrm{l}, \mathrm{p}=0.03)$ and similar to normal weight controls $(2.54 \pm 1.27 \mathrm{mcg} / \mathrm{l})$. Necdin and E2F4 gene expression in the adipose tissue were significantly down-regulated after GBS when compared with obese and were similar to levels observed in the lean controls. In human mature adipocytes cultured in vitro, treatment with desIGF-1 induced down-regulation of Necdin and E2F4 gene expression in a dose-dependent manner $(\mathrm{p}=0.01)$.

Conclusion-After RYGB, the insulin/IGF-1 signaling pathway is activated and could account for the observed decrease in expression of Necdin, which represses PPAR $\gamma$ promoter activity via interaction with E2F4. This could represent one of the mechanisms that induce resolution of insulin resistance status following RYGB.

() 2011 American Society for Bariatric Surgery. Published by Elsevier Inc. All rights reserved

Correspondence to: Alfonso Torquati MD, MSCI Associate Professor of Surgery Department of Surgery, Duke University 407 Crutchfield Street, Durham, NC 27704 alfonso.torquati@ duke.edu.

Publisher's Disclaimer: This is a PDF file of an unedited manuscript that has been accepted for publication. As a service to our customers we are providing this early version of the manuscript. The manuscript will undergo copyediting, typesetting, and review of the resulting proof before it is published in its final citable form. Please note that during the production process errors may be discovered which could affect the content, and all legal disclaimers that apply to the journal pertain.

Conflict of Interest: All the above authors do not have any conflict of interest with an institution or product that is mentioned in the manuscript and/or is important to the outcome of the study presented. 


\section{Keywords}

Necdin; E2F4; adipocyte; gastric bypass surgery; bariatric surgery; adipose tissue

\section{Introduction}

Adipose tissue plays an important role in modulating insulin sensitivity through production of metabolites, such as free fatty acids, and in its role as an endocrine organ producing a variety of adipokines ${ }^{(1)}$. In obesity, abnormal adipogenesis impairs insulin action and predisposes to type 2 diabetes mellitus (T2DM) In fact, the majority of patients with T2DM are obese or overweight ${ }^{(2)}$. In obese patients with T2DM, significant weight loss results in amelioration or resolution of their insulin resistance status. This increased insulin sensitivity results from insulin mediated enhancement of glucose uptake and from hepatic glucose output suppression ${ }^{(3)}$.

Previous studies have shown that Roux-en-Y gastric bypass (RYGB) is very effective in inducing remission of T2DM in morbidly obese patients ${ }^{(3)}$. Thus, one strategy for treating class II and III obese patients with T2DM who are not adequately treated by medical intervention is interventional surgery ${ }^{(4)}$. Defining the underlying mechanisms that cause the improvement after RYGB may potentially uncover novel molecular targets for non-surgical interventions. Towards this end, multiple studies have focused on identifying variables predictive of remission of T2DM following RYGB, including pre-operative BMI, HbA1c, visceral adiposity and duration of the diabetic status ${ }^{(5-6)}$.

Few studies have examined the role of insulin-like growth factor 1 (IGF-1) as a mediator of the anti-diabetic effects of bariatric surgery. Obesity is associated with the impairment of normal growth hormone $(\mathrm{GH})$ secretion, which regulates the production of IGF-1. IGF-1 levels significantly increase after surgical induced weight loss. Engström et al. ${ }^{(7)}$ investigated a well-characterized group of severely obese patients before and after RYGB. Serum GH and IGF-1 significantly increased 12 months after RYGB. It is well known that IGF-1 promotes adipocyte differentiation and thus increases insulin sensitivity and alters adipocyte differentiation through intricate signaling pathways ${ }^{(8)}$.IGF1 binding to its cell surface receptor stimulates phosphorylation of several intracellular IGF1 receptor substrates (IRSs) that lead to activate of the PI(3)K and the MAP kinase pathways. These insulin receptor substrate proteins regulate Necdin-E2F4 interaction that represses peroxisomeproliferator-activated receptor gamma (PPAR $\gamma$ ) transcription via a cyclic AMP response element binding protein (CREB)-dependent pathway ${ }^{(8)}$. PPAR $\gamma$ has been proven crucial in the control of terminal adipocyte differentiation, and is the target for insulin-sensitizing thiazolidinediones (TZDs), which are used clinically for treatment of $\mathrm{T}_{2} \mathrm{DM}^{(9-10)}$. Goldfine and others have demonstrated that changes in gene expression of E2F4 and Necdin after treatment with the selective PPAR $\gamma$ agonist rosiglitazone are linked to transformed adipocyte differentiation ${ }^{(11)}$.

Based on the evidences that the IGF-1 signaling pathway promotes adipocyte differentiation and therefore insulin sensitivity, we hypothesized that in obese patients RYGB induces activation of the insulin/IGF-1 signaling pathway that suppresses the expression of Necdin, which represses PPAR $\gamma$ promoter activity via interaction with E2F4. The aim of this study was to test the hypothesis that this pathway represents one of the mechanisms by which RYGB induces resolution of insulin resistance. 


\section{Methods}

\section{Subjects}

We carried out a prospective case-control study where two experimental groups (groups 1 and 2) were compared to a control group (group 3). Additional details regarding the three subject groups are included below:

Group 1: Non-obese subjects status post-RYGB (with a least 12 months follow up) undergoing an elective abdominal surgical procedure (e.g. cholecystectomy, abdominal wall hernia repair, and/or abdominoplasty; Group 2: Diabetic subjects with class II and III obesity undergoing RYGB; Group 3: Non-obese diabetic individuals (body mass index (BMI) 20-29) control subjects requiring an elective abdominal surgery. The study was approved by the institutional review board, and an informed, written consent was obtained from all subjects prior to participation. The study employed the following exclusion criteria: diagnosis of neoplastic disease, inflammatory bowel disease, acute cholecystitis, and pregnancy.

\section{Tissue sample preparation for recombinant extraction and quantitative RT-PCR}

During the surgical procedure, two $0.5-\mathrm{cm}^{3}$ biopsies of abdominal subcutaneous and intraabdominal omental fat tissue were obtained. The tissue samples were washed in PBS solution and then soaked in RNA later preservative solution (Qiagen, Courtaboeuf, France) and stored at $-80^{\circ} \mathrm{C}$ until analysis.

\section{RNA extraction and quantitative RT-PCR}

Total RNA was extracted from paired subcutaneous (SC) and omentum (OM) adipose tissue (100 mg tissue) using RNeasy Lipid Tissue Kit with an added treatment to remove genomic DNA using RNase-free DNase (Qiagen Inc., Valencia, CA). cDNA synthesis was carried out using SuperScript ${ }^{\mathrm{TM}}$ III Reverse Transcriptase (Invitrogen Corp., Carlsbad, CA) in the presence of random hexamers (Advantage; Clontech). Necdin and E2F4 gene expression were studied by quantitative, real time RT-PCR using the specific protocol for the iCycler iQ Detection System (BioRad, Hercules, CA, USA) with SYBR green fluorophore. The specificity of each amplification product was confirmed by melting curve profiles and agarose gel electrophoresis. All samples were run in duplicate and values averaged. The GAPDH gene was used as internal control to normalize the PCR reactions for the amount of RNA added. The data were processed using Gene Expression Analysis Software (Bio-Rad Life Science, Hercules, CA).

The following primers were designed and used for human Necdin Accession No. NM_002487.2 forward: 5'-TGTGGTACGTGCTGGTCAAGG-3' and reverse: 5'GGCGAGGATGAGGCTGGTG-3'; human E2F4 (Accession No.AF 527540) forward: 5'GCAGACCCCACAGGTGTTTT-3' and reverse: 5'-GACCACTCACACTCTTCAGG-3'; human GAPDH(Accession No.NM_002046) forward: 5'CCCATGT TCGTCATGGGTGT-3' and reverse: 5' TGGTCATGAGGTCCTTCCACGATA-3'. The PCR primer sequence for Necdin and E2F4 and GAPDH was chosen based on the sequences available in GenBank (www.ncbi.nlm.nih.gov/Genbank).

\section{IGF1 determinations by high-sensitivity enzymatic assay}

A fasting blood sample was also obtained from each subject immediately before the induction of general anesthesia, immediately spun in a refrigerated $\left(4^{\circ} \mathrm{C}\right)$ centrifuge at 3,000 $\mathrm{rpm}$ for 10 minutes and stored at $-80^{\circ} \mathrm{C}$ until analyzed. The extracted plasma was used for IGF1 determinations by high-sensitivity enzymatic assay (Active Non Extraction IGF-I IRMA, DSL-2800, Diagnostic Systems Laboratories, Inc.). 


\section{Cell culture and differentiation}

Cryopreserved human subcutaneous adipose stem cells (hASCs) purchased from Zen-Bio, (Zen-Bio Inc., RTP, NC) were maintained and differentiated according to the supplier's specifications. Differentiated adipocytes maintained in AM-1 medium (Zen- Bio Inc., RTP, NC) were exposed for 24 hours to 5, 10 and $100 \mathrm{ng} / \mathrm{ml}$ of des-(1-3)-IGF-I (des-IGF-I) (Diagnostic System Laboratories), then harvested by Trizol reagent (Invitrogen Corp., Carlsbad, CA). Des-IGF-1 was used in these experiments to avoid interactions with IGF binding proteins that normally sequester circulating IGF-1.

\section{Western blot analysis}

Whole-cell lysates were prepared using RIPA-Lysis buffer supplemented with protease (Roche Applied Science, Indianapolis, IN) and phosphate inhibitor cocktails (Sigma-Aldrich Corp., St. Louis, MO). The lysates were resolved on 4-12\% Bis-Tris-HCL buffered polyacrylamide gels (Invitrogen Corp., Carlsbad, CA) and transferred to polyvinylidene difluoride membranes. These membranes were blocked for an hour with $5 \%$ non-fat milk in TTBS buffer. Primary antibodies were diluted in 5\% non-fat milk Membranes were incubated with a primary antibody for $2 \mathrm{~h}$, after $1 \mathrm{~h}$ of blocking in PBS with $0.05 \%$ Tween 20 and $5 \%$ dry milk, and with primary antibodies for $1 \mathrm{~h}$ at room temperature.

Antibodies to Necdin NC243 (ABCAM) and anti-E2F4 (Santa Cruz Biotechnology) were used at 1:1000 and 1:200, respectively. Blots were incubated with horseradish peroxidaseconjugated anti-rabbit IgG (Cell Signaling Technologies) and visualized using chemiluminescence (Western Lightning ECL - PerkinElmer)

\section{Statistical Analysis}

Values are presented as the means \pm S.D., unless indicated otherwise. Data were analyzed by GraphPad Prism (Graphpad Software, La Jolla, CA). One way ANOVA (with Bonferroni's multiple $t$ test for post hoc analysis), paired or unpaired student's test were used for groups comparison as appropriate. Statistical significance was assumed for $\mathrm{p}$ value $<0.05$.

\section{Results}

A total of twenty six patients were included in this study as shown in Table 1 . There were no significant differences in term of mean age and sex distribution in the three groups. As shown in Figure 1, in the post-RYGB group free IGF-1 levels were significantly higher than the pre-RYGB obese patients $(2.55 \pm 1.54$ vs. $1.32 \pm 0.65 \mathrm{mcg} / \mathrm{l}, \mathrm{p}=0.03)$ and similar to nonobese controls $(2.54 \pm 1.27 \mathrm{mcg} / \mathrm{l})$.

Necdin and E2F4 expression levels were measured in RNA obtained from subcutaneous and omental fat tissue samples. As shown in Figure 2, in the post-RYGB Necdin and E2F4 gene expressions were found to be significantly lower than in the obese group and similar to non obese controls. Moreover, this significant change was observed in both the subcutaneous and omental fat tissue samples.

Studies in cultured differentiated adipocytes were consistent with results seen in adipose biopsies. Differentiated adipocytes were exposed for 24 hours to 5, 10 and $100 \mathrm{ng} / \mathrm{ml}$ desIGF-I followed by RNA analysis by Q-PCR. As shown in Figure 3, treatment of differentiated cultured adipocytes with increasing doses des-IGF-1 down-regulated Necdin and E2F4 gene expression in a dose-dependent manner ( $\mathrm{p}=0.01$ for $\mathrm{E} 2 \mathrm{~F} 4)$. These changes in gene expressions were confirmed at the protein level by Western blot analysis. 


\section{Discussion}

In our study, we have measured key regulators of insulin sensitivity in human plasma and adipose tissue samples following RYGB. Specifically, we focused on the role of IGF-1 and its downstream signaling in adipogenesis process because these pathways have previously been shown to play key roles in insulin sensitivity. A previous animal study determined that the IGF-1 signaling pathway promotes adipocyte differentiation and therefore insulin sensitivity via suppression of Necdin expression, which represses PPAR $\gamma$ promoter activity via interaction with $\mathrm{E} 2 \mathrm{~F} 4^{(8)}$. The aim of this study was to test the hypothesis that this pathway represents one of the mechanisms by which RYGB induces resolution of insulin resistance in obese patients. The changes we observed suggested Necdin-E2F4 interaction as one of the mediator for change in insulin sensitivity observed after RYGB. In specific, we propose a model whereby RYGB activates the insulin/IGF-1 signaling pathway which in turn up-regulates adipocyte differentiation via a Necdin-E2F4 signaling cascade in adipocytes. Our model is supported by the following observations: 1) Free IGF1 protein levels were significantly lower in obese diabetic patients versus lean counterparts; 2) Free IGF1 protein levels in obese patients increased to levels seen in the lean control population after RYGB; 3) Necdin and E2F4 mRNA levels were downregulated in subcutaneous and omental adipose cells following RYGB; and 4) in cultured human adipocytes, des-IGF-1 down-regulated Necdin and E2F4 gene expression in a dose-dependent manner. Other investigators have also observed improvements in glucose metabolism and increase in IGF-1 after RYGB ${ }^{(7)}$. These studies have focused on the fact that IGF1 stimulates production of an increased number of adipocyte precursor cells ${ }^{(7,12)}$.

Some of the conflicting results regarding IGF-1 levels in the literature may be due to differences in measuring free versus total IGF-1. One study reported that total IGF-1 concentrations were not significantly different between the obese and normal controls, despite GH hyposecretion in obesity ${ }^{(13)}$. But this same study also found that free IGF-1 concentrations were higher in obese subjects versus normal controls. Variations in total IGF-1 have also been seen to vary with factors such as ethnicity and age. For example, Caucasians who were obese in early childhood (increased BMI at age 21) had significantly lower total IGF1 levels compared to African Americans for those who were obese in early adulthood $^{(14)}$. In another study, total IGF-I was found to be significantly higher in lean adolescents than in obese adolescents, whereas basal insulin was elevated in obese adolescents compared to levels in both groups of lean subjects ${ }^{(15)}$. Though some studies have negatively correlated total IGF-I with obesity and intra-abdominal fat mass ${ }^{(16,17)}$, the observations in obese subjects are not consistent ${ }^{(18)}$. Circulating IGF-I interacts with IGF1 Binding Protein (IGFBP-1), and these together could be important determinants of glucose homoeostasis $^{(19)}$. For example, depressed levels of IGFBP-1 in obese individuals may affect levels of free IGF-1 bioactivity as well as its feedback regulation of GH secretion ${ }^{(20)}$. Further studies will be needed to correlate free IGF-1 and IGFBP-1 after RYGB.

We carried out experiments to directly support the association between IGF1 and NecdinE2F4 in differentiating human adipocytes. Our analysis showed that in human mature adipocytes, treatment with des-IGF-1 induces down regulation of Necdin and E2F4 gene expression in a dose-dependent manner. Our data are consistent with data reported by Tseng et $a^{(8)}$ after treatment of cultured adipocytes from mice. Necdin has been reported to act upstream of other regulators of adipogenesis. For example, knockdown of Necdin expression decreases expression of the adipogenesis inhibitors, Pref- 1 and Wnt10a, this restoring adipocyte differentiation. Additionally, Necdin has been shown to cooperate with the E2F4 family transcription factors that regulate PPAR $\gamma$ expression. Other studies have shown that IGF1 increases differentiation of cultured 3T3-L1 cells and pre-

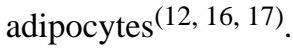




\section{Conclusion}

Our data suggested that activation of the insulin/IGF-1 signaling pathway after RYGB suppresses expression of Necdin, which represses PPAR promoter activity via interaction with E2F4. This study highlights this pathway as one of the mechanisms mediating the resolution of insulin resistance observed after RYGB. However, it is important to underscore that the changes we observed can be explained by the weight loss alone and being not specific to RYGB, considering that our study did not include a control group with a bariatric procedure without gastro-duodenal exclusion. To answer this question a future prospective study including other bariatric procedures should be considered. Understanding the key molecular changes that mediate the increased insulin sensitivity after bariatric procedures may lead to novel non-surgical therapies for type II diabetes in the future.

\section{Acknowledgments}

The study was supported by a National Institute of Health Grant: K23DK075907 (to A.T.)

\section{References}

1. Garg A. Regional adiposity and insulin resistance. J Clin Endocrinol Metab. 2004; 89:4206-10. [PubMed: 15356007]

2. Bays HE, Laferrère B, Dixon J, et al. Adiposopathy and bariatric surgery: is 'sick fat' a surgical disease? Int J Clin Pract. 2009; 63:1285-300. [PubMed: 19691612]

3. Bonhomme S, Guijarro A, Keslacy S, et al. Gastric bypass up-regulates insulin signaling pathway. Nutrition. 2011; 27:73-80. [PubMed: 21146129]

4. Lebovitz HE. Type 2 diabetes mellitus-current therapies and the emergence of surgical options. Nat Rev Endocrinol. 2011; 7:408-19. [PubMed: 21301486]

5. Hayes MT, Hunt LA, Foo J, Tychinskaya Y, Stubbs RS. A model for predicting the resolution of type 2 diabetes in severely obese subjects following Roux-en Y gastric bypass surgery. Obes Surg. 2011; 21:910-6. [PubMed: 21336560]

6. Torquati A, Lutfi R, Abumrad N, Richards WO. Is Roux-en-Y gastric bypass surgery the most effective treatment for type 2 diabetes mellitus in morbidly obese patients? J Gastrointest Surg. 2005; 9:1112-6. [PubMed: 16269382]

7. Edén Engström B, Burman P, Holdstock C, Ohrvall M, Sundbom M, Karlsson FA. Effects of gastric bypass on the GH/IGF-I axis in severe obesity--and a comparison with GH deficiency. Eur J Endocrinol. 2006; 154:53-59. [PubMed: 16381991]

8. Tseng YH, Butte AJ, Kokkotou E, et al. Prediction of preadipocyte differentiation by gene expression reveals role of insulin receptor substrates and Necdin. Nature Cell Biol. 2005; 7:543-5. [PubMed: 15928698]

9. Fan W, Morinaga H, Kim JJ, Bae E, et al. FoxO1 regulates Tlr4 inflammatory pathway signalling in macrophages. EMBO J. 2010; 29:4223-36. [PubMed: 21045807]

10. Olefsky JM, Glass CK. Macrophages. inflammation, and insulin resistance. Annu Rev Physiol. 2010; 72:219-46. [PubMed: 20148674]

11. Goldfine AB, Crunkhorn S, Costello M, et al. Necdin and E2F4 are modulated by rosiglitazone therapy in diabetic human adipose and muscle tissue. Diabetes. 2006; 55:640-50. [PubMed: 16505226]

12. Blüher S, Kratzsch J, Kiess W. Insulin-like growth factor I, growth hormone and insulin in white adipose tissue. Best Pract and Res Clin Endo Met. 2005; 19:577-87.

13. Nam SY, Lee EJ, Kim KR, et al. Effect of obesity on total and free insulin-like growth factor (IGF)-1, and their relationship to IGF-binding protein (BP)-1, IGFBP-2, IGFBP-3, insulin, and growth hormone. Int J Obes Relat Metab Disord. 1997; 21:355-9. [PubMed: 9152736]

14. Fowke JH, Matthews CE, Yu H, et al. Racial differences in the association between body mass index and serum IGF1, IGF2, and IGFBP3. Endocr Relat Cancer. 2010; 17:51-60. [PubMed: 19786462] 
15. Attia N, Tamborlane WV, Heptulla R, et al. The metabolic syndrome and insulin-like growth factor I regulation in adolescent obesity. J Clin Endocrinol Metab. 1998; 83:1467-71. [PubMed: 9589640]

16. Ramsay TG, White M, Wolverton CK. Insulin-like growth factor I - Induction of differentiation of porcine preadipocytes. J Animal Sci. 1989; 67:2452-9.

17. Blake WL, Clarke SD. Induction of adipose fatty acid binding protein by insulin-like growth factor I in 3T3-L1 preadipocytes. Biochem Biophys Res Comm. 1990; 173:87-91. [PubMed: 1701639]

18. Rasmussen MH, Frystyk J, Andersen T, Breum L, Christiansen JS, Hilsted J. The impact of obesity,fat distribution, and energy restriction on insulin-like growth factor-1 (IGF-1), IGFbinding protein-3, insulin, and growth hormone. Metabolism. 1994; 43:315-19. [PubMed: 7511202]

19. Sandhu MS, Heald AH, Gibson JM, Cruickshank JK, Dunger DB, Wareham NJ. Circulating concentrations of insulin-like growth factor-I and development of glucose intolerance: a prospective observational study. Lancet. 2002; 359:1740-5. [PubMed: 12049864]

20. Conover CA, Lee PD, Kanaley JA, Clarkson JT, Jensen MD. Insulin regulation of insulin-like growth factor binding protein-1 in obese and nonobese humans. J Clin Endocrinol Metab. 1992; 74:1355-60. [PubMed: 1375600] 


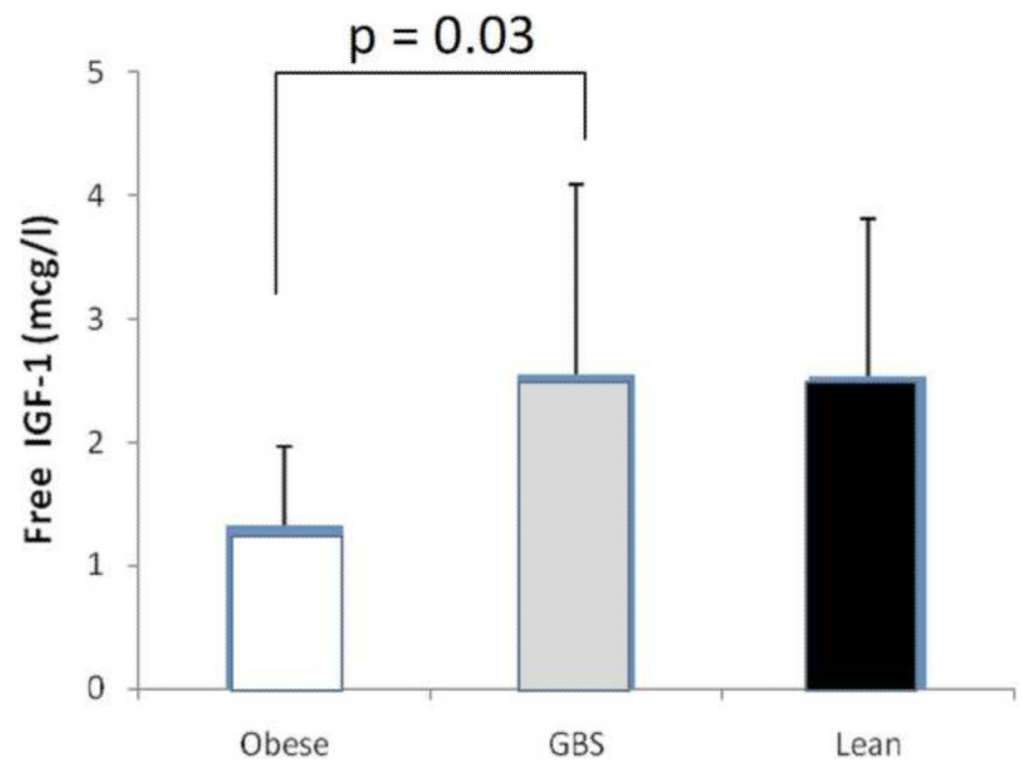

Figure 1.

Free IGF-1 levels were determined in plasma collected from obese patients (Obese), patients at least 12 months after RYGB, and non-obese controls (Lean). Data presented as mean \pm $1 \mathrm{SD}$. 

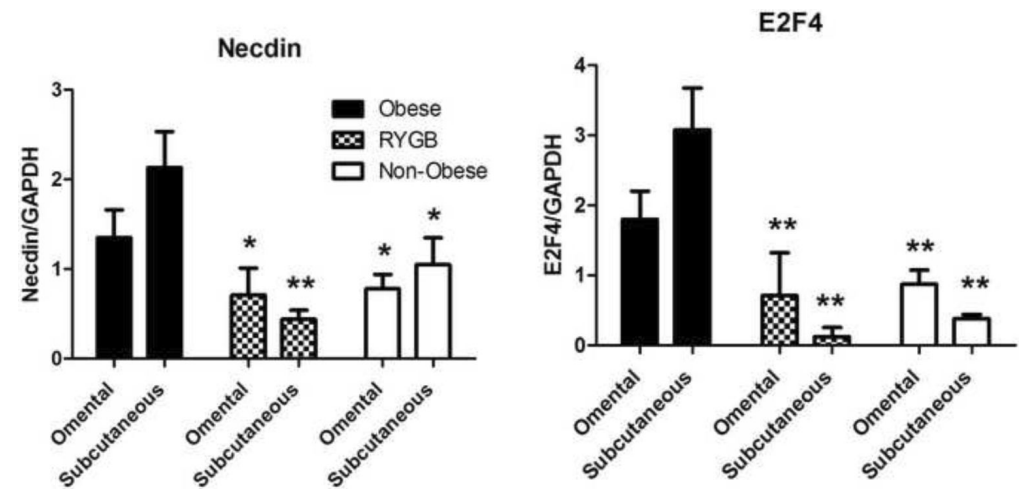

Figure 2.

Relative Necdin and E2F4 RNA levels normalized to GAPDH RNA levels in subcutaneous and omental adipose tissue collected from obese patients, patients at least 12 months after $\mathrm{RYGB}$, and non-obese controls. Data presented as mean $\pm \mathrm{SEM}$. $*=\mathrm{P}<0.05$ vs. obese; $* *=$ $\mathrm{P}<0.01$ vs. obese. 

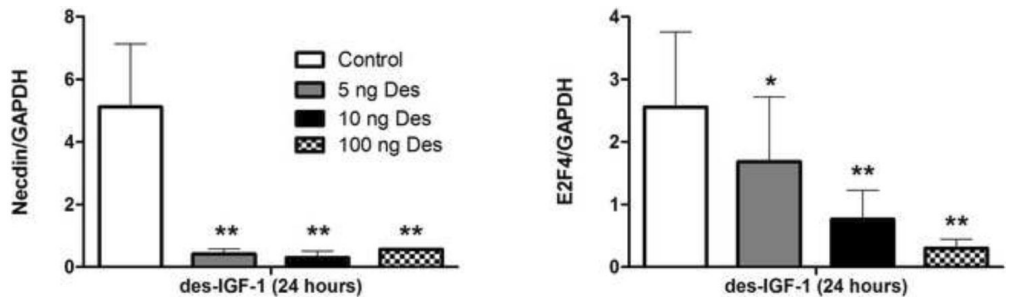

Figure 3.

Effect of des-IGF-1 on Necdin and E2F4 gene expression in cultured adipocytes. Data presented as mean \pm SEM. $*=\mathrm{P}<0.05$ vs. control; $* *=\mathrm{P}<0.01$ vs. control. 


\section{Table1}

Subject clinical characteristics

\begin{tabular}{lccc}
\hline Variable & Non-Obese Control & Obese & Post RYGB \\
\hline $\mathbf{n}$ & $\mathrm{n}=7$ & $\mathrm{n}=10$ & $\mathrm{n}=9$ \\
Gender (F/M) & $3 / 4$ & $7 / 3$ & $8 / 1$ \\
BMI $\left(\mathbf{k g} / \mathbf{m}^{\mathbf{2}}\right)$ & $27.6 \pm 2.8$ & $49 \pm 1.2$ & $25.6 \pm 2.8$ \\
Age(years) & $48 \pm 1.4$ & $40 \pm 9$ & $41 \pm 9$ \\
\hline
\end{tabular}

Values are mean \pm SD. 